\title{
Desejo, intenção e comportamento na saúde reprodutiva: a prática da cesárea em cidade do Nordeste do Brasil
}

\author{
Wishes, intention and behavior in reproductive health: \\ the practice of cesarean section in a city in the northeast of Brazil
}

Nilma Dias Leão Costa ${ }^{1}$, Neir Antunes Paes², Paulo César Formiga Ramos ${ }^{3}$, Maria Célia de Carvalho Formiga ${ }^{4}$

\section{RESUMO}

Objetivo: investigar os fatores determinantes da alta incidência do parto cesáreo e a sua inter-relação com a esterilização. Métodos: a pesquisa é parte de estudo multicêntrico sobre saúde reprodutiva no Brasil, realizado de 1998 a 2000, que incluiu os estados do Rio Grande do Norte, Minas Gerais, São Paulo e Rio Grande do Sul. Caracterizou-se como longitudinal prospectivo, no qual foram entrevistadas mulheres, provenientes do serviço público e do privado, em três momentos: no início da gravidez (até a 22 . semana de gestação), no final (entre 30 e 40 dias antes da data provável do parto) e após o nascimento do bebê (entre 15 e 45 dias pós-parto). As entrevistadas deveriam satisfazer aos critérios de elegibilidade: ter entre 18 e 40 anos e residir e ter o filho no município de Natal. Foram realizadas 433 entrevistas no primeiro momento, 380 no segundo e 269 no terceiro. Os dados foram submetidos ao teste $\mathrm{x}^{2}$ a uma significância de $\alpha=5 \%$, para comprovação da associação entre as variáveis anos de estudo (de 0 a 8 e 9 ou + ) e as variáveis representativas da saúde reprodutiva. Resultados: das entrevistadas que tiveram seguimento (269), 119 tiveram parto por cesárea (55\% do setor privado), sendo $45 \%$ previamente marcadas e $60 \%$ delas dias antes do parto. Os resultados revelaram relação estatisticamente significante $(\mathrm{p}<0,05)$ entre a escolaridade e as variáveis paridade, serviço procurado, classe social, estarem trabalhando e consultas de pré-natal. Assim, ficou demonstrado que a maior escolaridade predomina entre aquelas mulheres provenientes do serviço privado, de classe social mais elevada e com maior número de consultas de pré-natal. Embora sem significância estatística, observou-se para as mulheres desse serviço uma maior realização do parto cesáreo, provavelmente pela facilidade da interação entre o médico e a paciente, quando o desejo pela cesárea é frustrado para $43 \%$ daquelas provenientes do serviço público, bem como o desejo para fazer uma ligadura de trompas na hora do parto (57\%). Conclusões: esses resultados mostraram os grandes diferenciais existentes entre as categorias público e privado, demonstrando um claro favorecimento do setor privado, e que a prática obstétrica no Brasil, precisa mudar e melhorar, tanto entre aquelas mulheres com acesso ao serviço privado, que conseguem a realização da cesárea sem consistentes indicações médicas, quanto entre as mulheres com acesso ao serviço público, que enfrentam dificuldades para realização desse procedimento, mesmo com procedentes indicações médicas, de modo a proporcionar igualdade no direito reprodutivo dessas mulheres.

PALAVRAS-CHAVE: Cesárea; Saúde reprodutiva; Esterilização; Anticoncepção; Incidência; Estudos multicêntricos

\section{ABSTRACT}

Purpose: to generate knowledge in order to allow of the determination the factors affecting the high incidence of caesarean section and its relation to sterilization. Methods: the multicentric study on reproductive health in Brazil, carried out from 1998 to 2000, included the States of Rio Grande do Norte, Minas Gerais, São Paulo, and Rio Grande do Sul. It was characterized as a longitudinal study, where the women, proceeding from public as well as private health services, were interviewed at three moments: at the beginning of pregnancy (until the $22^{\text {nd }}$ week of gestation), at the end of pregnancy (between 30 and 40 days

1 Professora do Departamento de Saúde Coletiva da Universidade Federal do Rio Grande do Norte - UFRN - Natal (RN), Brasil; Pesquisadora do Grupo de Estudo Demográfico.

2 Professor do Departamento de Estatística da Universidade Federal da Paraíba - UFPA, Centro de Ciências Exatas e da Natureza. Campus I. Campus Universitário. João Pessoa (PB), Brasil.

3 Professor do Departamento de Estatística da Universidade Federal do Rio Grande do Norte - UFRN - Natal (RN), Brasil; Pesquisadores do Grupo de Estudo Demográfico.

4 Professor do Departamento de Estatística da Universidade Federal do Rio Grande do Norte - UFRN - Natal (RN), Brasil; Pesquisadores do Grupo de Estudo Demográfico.

Correspondência: Nilma Dias Leão Costa

Rua Graciliano Ramos 2899 - Capim Macio - 59080-070 - Natal - RN - Fone/Fax.Res. (0xx)(84) 3217-4324 Fax/Trab . (0xx) (84) 3215-4321

e-mail:nilma@ufrnet.br 
before the probable date of childbirth) and after delivery (between 15 and 45 days after childbirth). The interviewed women complied with the following eligibility criteria: to have 18 to 40 years of age, to live in Natal and plan to deliver the baby in Natal. At the first moment, 433 women were interviewed, 380 at the second moment and 269 at the third moment. The data were submitted to the chi-square test, with the level of significance set at 0.05 , just to assure the relation between the variable years of schooling ( 0 to 8 and 9 or + ) and the representative variables for reproductive health. Results: the result disclosed a statistically significant relationship $(p<0.05)$ between the variables: years of schooling, parity, type of service used, social class, job status, and prenatal consultations. It was found that a higher educational level is predominant among those women who searched for private services, coming from higher social classes and large number of prenatal visits. Although without statistical significance, having a for the women coming from this type of service, a higher number of caesarean section ${ }^{5}$ was observed, probably due to an easy doctor-patient interaction, while the planned caesarean section was frustrated for $43 \%$ of those women coming from public health services, as well as the wish to have a tubal ligation at childbirth (57\%). Conclusions: These results show up the great differentials between the private and public services, with a clear favoritism for the private sector, demonstrating clearly that the practical obstetrics in Brazil needs a change and an improvement both for those women with access to the private service, who obtain the accomplishment of the caesarean section without consistent medical indications, and those with access to the public service, who face difficulties in accomplishing this procedure, even with consistent medical indications, in order to provide equality in the reproductive rights of those women.

KEYWORDS: Caesarean section; Reproductive medicine; Sterilization; Contraception; Incidence; Multicenter studies

\section{Introdução}

No Brasil, desde a década de 80, muitos estudos se dedicavam a questões da saúde reprodutiva como uma das formas de modificar a fecundidade. A Pesquisa sobre Saúde da Família no Nordeste do Brasil, realizada em 1991, pela Sociedade Civil Bem-Estar Familiar no Brasil - BEMFAM ${ }^{1}$, mostrou que a taxa de fecundidade total - TFT, na faixa etária entre 15 e 49 anos, foi de 2,8 filhos, na zona urbana, e de 5,2 filhos, na zona rural, sendo de 3,7 filhos por mulher na Região Nordeste. No Brasil essa taxa encontra-se em declínio, porém ainda distante do nivel de reposição populacional, que é de 2,1 filhos por mulher.

A confirmação desse declínio pode ser constatada, também, por meio da Pesquisa Nacional sobre Demografia e Saúde - PNDS $/ 96^{2}$, realizada pela BENFAM, que mostra uma TFT de 2,5 e 3,1 filhos por mulher, para o Brasil e para região Nordeste, respectivamente. Os fatores associados a esse declínio estariam relacionados à autonomia, à autodeterminação das mulheres e, também, ao conhecimento e à utilização de algum método anticoncepcional por grande parte delas, pois o homem brasileiro, praticamente, ainda não participa com nenhum método contraceptivo ${ }^{3,4}$.

Sabe-se que o método contraceptivo mais utilizado é a pílula. Porém, outro que tem se destacado é a esterilização feminina como prática comum dentre os muitos métodos. Além disso, muitos estudos apontam a esterilização feminina como um dos fatores determinantes da queda da fecundidade, observando que ela ocorreu, primeiramente, entre mulheres de classe social mais elevada, com maior escolaridade e moradoras da zona urbana ${ }^{5,6}$.
Investigação sobre a contracepção e a queda da fecundidade mostrou que esse declínio aconteceu também entre as mulheres socialmente menos privilegiadas, moradoras de zona rural e com menor grau de instrução ${ }^{5}$. O estudo revelou que não existe diferença socioeconômica em relação à esterilização e que este método tem sido mais usado por mulheres jovens, com menos filhos ou baixa parturição. Ele também apontou como fatores redutores da fecundidade o padrão de casamento, a infertilidade e o aborto. Sugere, enfim, novos estudos que possibilitem uma investigação mais sistemática nesse sentido.

A esterilização, adotada como forma de contracepção no país, demonstra a omissão do setor de saúde pública quanto a um planejamento familiar eficaz, permitindo a implantação de programas para controle da natalidade através de órgãos internacionais não governamentais ${ }^{3,4}$.

No Brasil, em 1997, segundo a Pesquisa Nacional em Demografia e Saúde ${ }^{1}$, a esterilização chegou a um patamar de $42,0 \%$ como método contraceptivo, sendo que, para o Nordeste, esse percentual foi de $43,0 \%$ e, segundo o censo demográfico de 1991, foi de $44,0 \%$. São preocupantes as informações que se referem à esterilização feminina como sendo fenômeno que ocorre em maior proporção entre mulheres ainda jovens, na faixa etária de 25 a 30 anos. Normalmente, ela é adotada em maior escala a partir do segundo filho, com a mulher na idade de 30 anos e realizada durante o parto cesáreo ${ }^{3}$.

Dados nacionais confirmam a tendência de crescimento desse tipo de parto, que alcançou $36 \%$ no Brasil, com taxas maiores em São Paulo (52\%) e na Região Centro-Oeste (49\%), sendo as menores taxas nas Regiões Nordeste $(20 \%)$ e Norte $(25 \%)$. Observa-se, ainda, que o parto cesáreo é mais fre- 
qüente na região urbana $(41,8 \%)$ do que na região rural $(20,1 \%)$. Esta prática mostra-se também fortemente associada à condição social, especialmente ao grau de instrução da mulher, aumentando progressivamente com o número de anos de estudo ${ }^{2}$.

A proporção de partos cesáreos é maior em mulheres adultas, mas é considerado elevado para a faixa etária de adolescentes atendidas pelo SUS. Os problemas mais freqüentes que levam à decisão da cesárea são aqueles relacionados às intercorrências obstétricas, como o parto pré-termo, as síndromes hipertensivas e a desproporção fetopélvica. Esse estudo realizado em Ribeirão Preto ${ }^{7}$ concluiu que na faixa da população mais jovem, com poder econômico baixo, com menos escolaridade e usuária do serviço público, ainda há carência de medidas preventivas que possam contribuir para o declínio da gravidez na adolescência.

Outro fator fortemente associado a um maior risco de levar a um parto cesáreo é a questão do parto de repetição. Este fato leva a propor medidas que possam contribuir para melhorar o indice do parto normal após cesárea, por meio da realização da prova do trabalho de parto (prática rara nas escolas médicas), o uso de analgesia para aliviar a dor na hora do parto e critérios que possam esclarecer melhor o diagnóstico de sofrimento fetal ${ }^{8}$. O medo pelo parto normal acontece e é visivel, quando a razão principal para levar a um parto cesáreo é a dor. A falta de informação, de um diálogo que possa trazer tranqüilidade numa conversa franca e esclarecedora entre profissionais de saúde e paciente poderia melhorar os anseios da mulher em relação ao parto. Por isso, é necessário "mudança de atitudes dos procedimentos obstétricos", para que haja melhor prática do conhecimento nos "procedimentos médicos".

Resultados do estudo multicêntrico sobre saúde reprodutiva no Brasil para o conjunto dos estados envolvidos (São Paulo, Rio Grande do Sul, Minas Gerais e Rio Grande do Norte) revelaram que tem sido excessiva a prática de partos operatórios com predomínio da contracepção cirúrgica, constituindo-se, dessa forma, como um problema de saúde pública, afetando não só a morbimortalidade reprodutiva, mas também as condições do recémnascido e os custos hospitalares para o sistema previdenciário, que crescem desnecessariamente ${ }^{10}$.

Com isso, o presente trabalho tem como propósito estudar os fatores que contribuem para essa ainda elevada utilização de partos cesáreos, que vão além dos motivos de indicação médica para a mãe ou para o feto e levantam razões para uma investigação. O que se deseja é procurar conhecer, entre as entrevistadas na cidade de Natal, RN, as razões sociais e/ou médicas que fazem estas mu- lheres ou profissionais de saúde decidirem por esse tipo de parto, pela contracepção cirúrgica, como também suas conseqüências e implicações.

\section{Métodos}

O trabalho caracterizou-se como estudo multicêntrico, longitudinal e prospectivo, desenvolvido entre abril de 1998 e junho de 2000, no qual as gestantes foram acompanhadas desde o momento em que procuraram um serviço de saúde para realização do pré-natal até o pós-parto. Esse estudo incluiu as regiões metropolitanas de Belo Horizonte, São Paulo, Porto Alegre e Natal, sob a coordenação central da Universidade do Texas, USA. Para efeito da discussão que se propõe neste artigo, a base utilizada contará apenas com as entrevistas realizadas em Natal, RN.

A cidade de Natal é capital do Rio Grande do Norte, situada na Região Nordeste do Brasil. De acordo com o censo 2000, possui uma população total de 709.422 (376.726 homens e 332.696 mulheres). Natal representa uma cidade típica do Nordeste. Apresenta indicadores de saúde que mostram ainda uma necessidade de investimento que possa melhorar a saúde da sua população. Segundo dados do SINASC ${ }^{11}$ (2002-2003), a mortalidade infantil ainda chega a $23,8 \%$ o nascidos vivos. A mortalidade materna apresenta-se em torno de $71,2 \%$ o nascidos vivos. Quanto à escolaridade das mães, 50,3\% têm menos de 8 anos de estudo. Antecedentes reprodutivos mostraram que $47,4 \%$ delas tem de 1 a 3 filhos, 16,1\% realizaram menos de 4 consultas de pré-natal e o parto cesáreo foi indicado para $36,0 \%$ das mulheres como forma "normal" para o nascimento do seu filho.

O acompanhamento à gestante foi realizado por meio da aplicação de três entrevistas em momentos distintos: no momento da consulta de pré-natal (estando a gravidez até a $22^{\mathrm{a}}$ semana), na residência da gestante (30 a 40 dias antes da data provável do parto) e após o nascimento da criança (entre 15 e 45 dias pós-parto).

O estudo satisfez a um critério de elegibilidade, abrangendo uma amostra das mulheres de 18 a 40 anos de idade que residiam em Natal, estavam grávidas no máximo na $22^{a}$ semana de gestação e procuraram o serviço público ou privado para realização do pré-natal. O tamanho amostral teve por base a conveniência em vez de critérios probabilísticos, tendo em vista a limitação dos recursos disponiveis e o elevado custo de um estudo prospectivo com três momentos de entrevista. Esse esquema amostral foi baseado no SINASC para a distribuição das respectivas gestantes na categoria 
de primipara e multipara, distribuída por setor de atendimento, público e privado. Ao todo foram realizadas 433 entrevistas no primeiro momento (Q1), 380 no segundo (Q2) e 269 no terceiro (Q3). Essas diferenças quantitativas são justificadas por entrevistas perdidas e por razões como a perda do bebê e a mudança de endereço da entrevistada.

O estudo foi aprovado no Comitê de Ética da Universidade Federal do Rio Grande do Norte e nos demais comitês de cada estado. O consentimento informado foi assinado pela entrevistada e concedido à utilização das informações para cunho científico.

Os questionários foram codificados e a entrada dos dados foi feita pelo do software Epi-Info 6.04. Para análise de associações entre todas as condições (variáveis), foram utilizado os softwares SPSS 12.0 e o STATISTICA 6.0.

A proposta desse estudo foi realizar estudo descritivo dos resultados e analisar o comportamento das variáveis e indicadores nos três momentos da pesquisa. Utilizou-se o teste $\mathrm{x}^{2}$ para investigar a existência de associação entre algumas variáveis referentes à saúde reprodutiva da mulher e a variável educação, estabelecendo-se nível de significância de $5 \%(\mathrm{p}<0,05)$. Essa variável foi selecionada tendo em vista a sua importância nas questões ligadas à saúde da população e por ser proxi de condição social.

Já a variável socioeconômica foi classificada em cinco categorias (A, B, C, D e E), segundo o novo critério Brasil adotado pela Sociedade Brasileira de Pesquisa de Mercado - SBPM ${ }^{12}$, que associa valores (pesos) ao número de bens de consumo declarado pelo entrevistado, pontuando, também, o grau de instrução do chefe da família, com estratos que vão da situação mais favorecida (A) à menos favorecida (E).

\section{Resultados}

\section{Perfil social das entrevistadas}

Nos três momentos da pesquisa $(\mathrm{Q} 1, \mathrm{Q} 2$, e Q3), em relação ao tipo de serviço utilizado, observou-se predominância do serviço público, uma vez que, nas três etapas do estudo, o percentual correspondente de mulheres entrevistadas em $\mathrm{Q}_{1}, \mathrm{Q}_{2} \mathrm{e}$ $\mathrm{Q}_{3}$ foi, respectivamente, 72,68 e $68 \%$. No primeiro momento da pesquisa, a população do estudo foi formada por 433 mulheres, cuja distribuição por parturição se deu em 53\% multíparas (por já ter realizado um ou mais partos), predominando um atendimento pelo SUS (57\%). Dentre as primíparas (por se tratar do primeiro parto), o maior atendimento foi feito pelo serviço privado (56\%).

Verificou-se, na amostra de 433, que a maior incidência de mulheres grávidas ocorreu na faixa etária de até 25 anos, representando, aproximada- mente, $52 \%$ do total, dessas, $13 \%$ encontravam-se na faixa de 18 a 19 anos. Em relação as mulheres adultas (aquelas maiores de 20 anos), a maioria $(72 \%)$ encontravam-se entre 20 e 30 anos de idade. A maioria se autodenominou não-branca (64\%), sendo predominantemente casada (92\%), tendo escolaridade razoável $(49 \%$ das gestantes com 9 ou + anos de estudo), porém, 13\% apresentando menos de 4 anos de estudo.

A categorização das entrevistadas por classe social ou estrato social só foi possivel para 267 mulheres, que responderam às questões referentes a tal classificação, segundo o novo critério Brasil - (SBPM, 1997) ${ }^{12}$. Houve predominância de mulheres entrevistadas nas classes D e E (46\%), o que se mostra coerente com a maior proporção de mulheres que tiveram o parto pago pelo SUS (68\%).

\section{Perfil da saúde reprodutiva das entrevistadas}

Do total de mulheres entrevistadas (433), 53\% fizeram suas entrevistas até o $3^{\circ}$ mês de gestação, o que, para a pesquisa, tinha um forte significado, uma vez que para a gestante fazer parte do estudo precisava ter tido um número limitado de consultas ou de contato com o médico que a acompanhava. Em 33\% dos casos, as gestantes estavam realizando sua primeira consulta no momento da entrevista. Na realização do Q2 (entrevista realizada até alguns dias antes do parto), aproximadamente $72 \%$ das entrevistadas já tinham ido ao médico mais de cinco vezes, o que representou número médio de consultas de 2,07 e desvio padrão de 0,79.

Quando questionadas sobre o planejamento da gravidez, $60 \%$ das mulheres responderam que não tinham a pretensão de engravidar naquele momento, não tendo, portanto, planejado aquela gestação. Número expressivo de mulheres externou o desejo de querer ter o filho por parto normal (69\%). A conversa com o médico sobre tipos de parto foi constatada entre $14 \%$ das entrevistadas e dessas foi sugerido o parto do tipo cesariana para $45 \%$. Em $23 \%$ dos casos, o médico aconselhou que deveriam aguardar mais um pouco para posterior decisão. Ao serem indagadas acerca da razão mais importante para preferirem o parto normal, 30\% responderam que a sua recuperação seria mais rápida, e das que preferiram parto cesáreo, $12 \%$ pretendiam fazer ligadura tubária (LT). Entre aquelas mais jovens ( $<20$ anos), a preferência pelo parto normal foi também por levar em consideração a recuperação ser mais rápida e por não ser uma cirurgia. Nesta faixa etária, não se constatou nenhuma preferência por cesárea.

Dentre as 119 mulheres submetidas ao parto cesáreo, apenas 46 (64\%) souberam informar a razão 
do procedimento. Os motivos que as levaram à decisão por esse tipo de parto foram os mais diversos, podendo-se destacar a má apresentação do feto, em $27 \%$, sofrimento fetal, em $16 \%$, e $11 \%$ por problemas prévios da gravidez. Observou-se também que as mulheres entre 18 e 30 anos de idade (64\%) eram as que mais desejavam ter o parto normal, constatando-se que a maioria delas $(60 \%)$ teve seu desejo realizado. No grupo de mulheres mais jovens (18 a 19 anos), predominou o parto normal (74\%). Por sua vez, entre aquelas acima de 30 anos de idade, houve preferência pelo parto cesáreo (69\%).

No que se refere ao desejo de fazer esterilização, este foi observado tanto no Q1 (433) e Q2 (380), como contabilizado no Q3 (269), em torno de $21 \%$. É importante destacar, nessa questão, que $59 \%$ das entrevistadas não pretendiam mais ter filhos e que existiu demanda frustrada da esterilização em torno de $61 \%$ das mulheres. Ou seja, das 72 mulheres que pretendiam fazer a esterilização, $28(39 \%)$ conseguiram que o procedimento fosse efetuado e, dessas, $57 \%$ eram mulheres atendidas pelo serviço público. Contudo, verificou-se para essas mulheres elevado nível de frustração, não só em relação ao desejo de cesárea, como também o de esterilização, pois, para elas, a demanda frustrada pela esterilização atingiu o percentual de $87 \%$.

É interessante ressaltar que, quando indagadas se estavam satisfeitas por terem tido o bebê por cesárea (119 casos, tanto pelo serviço público como pelo privado), observaram-se relatos de satisfação ou insatisfação, detectando-se mais insatisfação naquelas provenientes do setor privado (71\%). Com relação à concordância com o procedimento médico, foram também as mulheres atendidas pelo serviço privado que expressaram maior grau de satisfação (55\%).

Das 269 mulheres que tiveram seguimento no estudo, $74 \%$ não tomaram medicamento algum para dor, independente do sexo e da parturição, o que poderá trazer conseqüências para uma próxima decisão do tipo de parto, quando leva a optar precipitadamente por um parto cesáreo como forma de evitar a dor. Entre aquelas que tiveram um parto anterior (81), 72\% não tomaram medicamento nenhum para dor e foram submetidas ao parto normal. Dessa forma, a proteção medicamentosa para dor foi administrada apenas para $16 \%$ das mulheres por ocasião da realização do parto normal. Dentre as mulheres submetidas ao parto normal (174), o trabalho de parto foi intensificado com ocitocina em 109 , das quais $79 \%$ eram provenientes do SUS. Porém, quando se observou o uso da ocitocina por tipo de serviço, perceberam-se percentuais semelhantes, 63 e $61 \%$, para o SUS e serviço privado, respectivamente. O tempo de espera da sua admissão no serviço até a realização do parto foi em média 4,7 horas, variando este tempo de espera de zero a trinta horas entre aquelas do serviço público. Naquelas do serviço privado, este tempo de espera foi em média, 2,5 horas, variando de 1 a 7 horas, demonstrando maior rapidez de atendimento para este grupo.

Entre as 269 mulheres acompanhadas nos três momentos do estudo, $88 \%$ externaram o desejo de não ter mais filhos. Para evitar a concepção, $32 \%$ declararam a intenção de fazer LT. Das que desejavam fazer LT, $57 \%$ eram provenientes do serviço público. O método anticoncepcional mais citado pelas mulheres foi a pílula, representando $24 \%$, seguido do DIU, $13 \%$. Os demais métodos apresentaram-se com menos de $20 \%$.

\section{Relação entre anos de estudo com variáveis associadas à vida Reprodutiva}

A variável educação, dada a sua importância para as questões associadas à saúde da população, foi selecionada para uma análise mais particularizada, investigando-se sua associação com algumas variáveis associadas à vida reprodutiva da mulher. Aplicou-se o teste $\mathrm{x}^{2}$, no intuito de certificar-se da associação entre a variável anos de estudo e as variáveis representativas da saúde reprodutiva (parturição, tipo de serviço, estrato social, estar trabalhando, tipo de parto desejado, número de cesáreas anteriores, marcação da cesárea, motivo pelo qual a cesárea tinha sido marcada, número de consultas de pré-natal, pretensão de LT, filhos vivos, como pretende evitar filhos e se fez LT). Fez-se categorização para anos de estudo, estabelecendo-se as categorias de 0 a 8 e de 9 ou +. Utilizou-se, para esta análise com a variável escolaridade, apenas o total de mulheres submetidas ao parto cesáreo (119).

Em virtude de o objetivo do estudo estar direcionado para verificar a prática da cesárea que vão além dos motivos de indicação médica para a mãe ou para o feto; também se deseja conhecer as razões sociais que fazem a mulher decidir por esse tipo de parto, suas conseqüências e implicações.

As variáveis demográficas e reprodutivas apresentadas na Tabela 1 e correlacionadas com anos de estudo ( 0 a 8 e de 9 ou + ) mostraram resultado significativo, com nivel de significância $a=5 \%$, no que se refere às variáveis parturição, tipo de serviço procurado, classe social, estar trabalhando e consultas de pré-natal. As demais variáveis não se mostraram significativas nessa população estudada, provavelmente em função do elevado número de mulheres primiparas (61\%), bem como do igualmente elevado número $(78 \%)$ de entrevistadas que não tinham sido submetidas a nenhum parto cesáreo.

A associação entre paridade e escolaridade foi significante $(\mathrm{p}<0,05)$. No entanto, mesmo não tendo 
Tabela 1 - Distribuição das entrevistadas segundo as categorias de anos de estudo e as demais variáveis socioeconômicas, demográficas e reprodutivas.

\begin{tabular}{|c|c|c|c|}
\hline \multirow{2}{*}{ Perfil das gestantes } & \multicolumn{2}{|c|}{ Anos de estudo } & \multirow{2}{*}{$\mathrm{p}$} \\
\hline & 0 a $8(\%)$ & 9 ou $+(\%)$ & \\
\hline \multicolumn{4}{|l|}{ Parturição } \\
\hline Primípara & $14(44)$ & $59(68)$ & \multirow{3}{*}{0,02} \\
\hline Multípara & $18(56)$ & $28(32)$ & \\
\hline $\mathrm{N}$ & $32(100)$ & $87(100)$ & \\
\hline \multicolumn{4}{|l|}{ Tipo de serviço } \\
\hline SUS & $25(78)$ & $26(30)$ & \multirow{3}{*}{0,00} \\
\hline Privado & $7(22)$ & $61(70)$ & \\
\hline $\mathrm{N}$ & $32(100)$ & $87(100)$ & \\
\hline \multicolumn{4}{|l|}{ Classe social } \\
\hline$A+B$ & $0(0)$ & $36(41)$ & \multirow{3}{*}{0,00} \\
\hline$C+D+E$ & $31(100)$ & $51(59)$ & \\
\hline $\mathrm{N}$ & $31(100)$ & $87(100)$ & \\
\hline \multicolumn{4}{|l|}{ Está trabalhando } \\
\hline Sim & $8(25)$ & $56(64)$ & \multirow{3}{*}{0,00} \\
\hline Não & $24(75)$ & $31(36)$ & \\
\hline $\mathrm{N}$ & $32(100)$ & $87(100)$ & \\
\hline \multicolumn{4}{|l|}{ Tipo de parto desejado ${ }^{1}$} \\
\hline Parto normal & $21(68)$ & $57(70)$ & \multirow{3}{*}{0,79} \\
\hline Cesárea & $10(32)$ & $24(30)$ & \\
\hline $\mathrm{N}$ & $31(100)$ & $81(100)$ & \\
\hline \multicolumn{4}{|l|}{ Número de partos cesáreos² } \\
\hline Nenhum & $7(39)$ & $13(46)$ & \multirow{3}{*}{0,62} \\
\hline $1 \mathrm{ou}+$ & $11(61)$ & $15(54)$ & \\
\hline $\mathrm{N}$ & $18(100)$ & $28(100)$ & \\
\hline \multicolumn{4}{|l|}{ Marcação da cesárea } \\
\hline Sim & $15(47)$ & $38(44)$ & \multirow{3}{*}{0,76} \\
\hline Não & $17(53)$ & $49(56)$ & \\
\hline $\mathrm{N}$ & $32(100)$ & $87(100)$ & \\
\hline Motivo pelo qual a cesárea foi $m$ & & & \\
\hline Problemas gravidez atual & $5(33)$ & $15(39)$ & \\
\hline Cesárea anterior & $2(13)$ & $1(3)$ & 0.51 \\
\hline Possibilidades De LT & $1(7)$ & $3(8)$ &, 01 \\
\hline Outro & $7(47)$ & $19(50)$ & \\
\hline $\mathrm{N}$ & $15(100)$ & $38(100)$ & \\
\hline Consultas de pré-natal & & & \\
\hline 1 a 5 & $11(37)$ & $11(13)$ & \\
\hline 6 a 7 & $12(40)$ & $36(43)$ & 0,01 \\
\hline $8 \mathrm{ou}+$ & $7(23)$ & $36(43)$ & \\
\hline $\mathrm{N}$ & $30(100)$ & $83(100)$ & \\
\hline Quando pretende fazer $\mathrm{LT}$ & & & \\
\hline Não sabe & $1(8)$ & $3(16)$ & 060 \\
\hline Junto com parto & $12(92)$ & $16(84)$ & 0,00 \\
\hline $\mathrm{N}$ & $13(100)$ & $19(100)$ & \\
\hline Fez LT & & & \\
\hline Sim & 10(31) & $18(21)$ & 023 \\
\hline Não & $22(69)$ & $69(79)$ & 0,23 \\
\hline N & $32(100)$ & $87(100)$ & \\
\hline Filhos Vivos ${ }^{2}$ & & & \\
\hline$\leq 1$ & $14(78)$ & $22(79)$ & 005 \\
\hline $2 \mathrm{ou}+$ & $4(22)$ & $6(21)$ &, 90 \\
\hline $\mathrm{N}$ & $18(100)$ & $28(100)$ & \\
\hline Como Pretende evitar filhos & & & \\
\hline Não sabe & $4(12)$ & $20(23)$ & \\
\hline Usar pilula & $6(19)$ & $21(24)$ & \\
\hline Colocar DIU & $3(9)$ & $10(12)$ & 0,49 \\
\hline Fazer LT & $1(38)$ & $21(24)$ & \\
\hline Outros & $7(22)$ & $15(17)$ & \\
\hline $\mathrm{N}$ & $3(100)$ & $87(100)$ & \\
\hline
\end{tabular}


significância $(p>0,05)$ a questão de "partos anteriores por cesárea", observou-se que, dentre aquelas que tiveram um ou mais filhos por cesárea, elas tinham um maior nivel de escolaridade (58\%).

Isso pode ser reforçado em relação às questões sociais, quando se obteve significância estatística para as variáveis relativas ao serviço procurado, classe social e o fato de estar ou não trabalhando. Constatou-se dessa forma a importância da maior escolaridade ter predominado entre aquelas do serviço privado, de classe social mais elevada e de estarem trabalhando no momento do parto atual.

Quanto à questão "tipo de parto desejado", constatou-se que não existe preferência por determinado tipo de parto relacionado com anos de estudo. Das 87 gestantes com 9 anos ou mais de estudo, $44 \%$ já haviam marcado a cesárea antes de entrar no hospital, contra apenas $15(47 \%)$ das que tinham até 8 anos de estudo. Embora, sem significância estatística, essa relação sugere maior facilidade de interação entre médico e paciente para este entendimento prévio.

Das 53 mulheres que marcaram a cesárea antecipadamente, 38 (44\%) tinham 9 anos ou mais de estudo e responderam que apresentavam problemas com a gravidez atual. Esses problemas eram devidos a questões como a posição do bebê, apresentação anômala ou condição inadequada. A utilização da cesárea como possibilidade para ligadura de trompas foi externada por 4 entrevistadas, a maioria com 9 anos ou mais de estudo (8\%).

Sabendo-se que a assistência pré-natal tem como objetivo principal garantir a evolução normal da gravidez e, dentre outros, identificar as situações de risco que venham a complicar este estado, pode-se observar que houve maior percentagem de grávidas que fizeram 8 consultas de pré-natal entre aquelas que tinham 9 ou mais anos de estudo (43\%). Ficou visivel, em qualquer número de consultas, a predominância por acompanhamento de pré-natal por aquelas com um grau de instrução mais elevado. Portanto, à medida que aumenta o grau de escolaridade também aumenta o número de consultas de pré-natal, $(p<0,05)$. Esse fato revela a importância, para a mulher, de um nivel educacional que se reverte em maiores cuidados de pré-natal e em sua vida reprodutiva, de um modo geral. Trata-se portanto de fator determinante para a queda da morbimortalidade materno-infantil.

A LT foi desejada por 28 entrevistadas que pretendiam realizá-la junto ao parto. Dessas, 43\% eram pacientes do SUS e tiveram seu desejo frustrado. Não foi observada significância estatística na relação entre essa variável e a escolaridade, uma vez que, das 32 mulheres que desejavam a LT, tanto entre as de baixa ( 0 a 8 anos de estudo) quanto entre as de alta escolaridade ( 9 anos ou mais), o desejo expressado de realizar a LT junto ao parto foi 92 e $84 \%$, respectivamente. Poucas mulheres, em ambas as categorias, não sabiam quando iriam ser submetidas ao procedimento. Dentre as submetidas à cesárea, a esterilização foi frustrada para $4(57 \%)$ das entrevistadas provenientes do SUS.

O número de filhos vivos, reportado pelas mulheres submetidas à cesárea (119), não variou significativamente com o grau de escolaridade $(p>0,05)$, especialmente pela predominância daquelas com menos de um filho e com mais anos de estudo (79\%), mostrando, dessa forma, uma possivel associação entre o grau de instrução e o número de filhos vivos.

Das entrevistadas que foram submetidas ao parto cesáreo, 28\% pretendiam evitar filhos fazendo LT, seguido do uso da pílula $(23 \%)$ e do DIU (11\%). É importante ressaltar que 20\% das mulheres não sabiam, ainda, como iriam evitar filhos. Apesar de os resultados não terem mostrado significância estatística no que diz respeito à relação entre escolaridade e escolhas contraceptivas $(p>0,05)$, eles revelaram uma preferência das mulheres com maior escolaridade ( 9 anos ou mais de estudo) tanto por métodos mais modernos como por tradicionais, haja vista que a opção pelo uso da tabela e da camisinha só foi reportada dentre esse grupo de mulheres $(100 \%)$, seguindo-se da preferência pelo uso da pílula (24\%), do DIU (12\%) e da LT (24\%). Assim, pode-se dizer que a escolaridade da mulher influencia fortemente nas suas opções contraceptivas e, na verdade, parece ser o fator motivador da concretização dessas opções.

A decisão pela indicação do parto cesáreo, para $63 \%$ das entrevistadas com razoável escolaridade, não contou com a sua participação ou de qualquer membro da família.

\section{Discussão}

A amostra do estudo foi composta por 433 mulheres, com considerável percentual entre as multiparas do SUS (57\%) e primiparas do serviço privado (56\%). Predominaram entre elas aquelas de idade adulta, consideradas de 20 a 30 anos de idade, com $72 \%$. A escolha do parto pelas mais jovens (18 a 19) anos foi pelo parto normal, ao passo que as adultas de (20 a 30 ou de 30 ou + ) preferiram o parto cesáreo ${ }^{7}$.

Das 269 mulheres que tiveram seguimento, $83(31 \%)$ tiveram apenas um parto anterior e 
dessas, $23 \%$ foram submetidas a uma cesárea. Quando se investigou esse grupo de mulheres com uma cesárea anterior (19), observou-se que 95\% (18) tiveram parto atual por cesárea. Este fato põe em evidência a cultura de "cesárea, sempre cesárea"8.

Quando se investigou a relação entre saúde reprodutiva e a variável anos de estudo, observouse, inicialmente, que há forte associação dessa variável com a parturição, sendo majoritário o número de primiparas $(81 \%)$ entre as mulheres de maior escolaridade ( 9 anos ou mais de estudo). Igual intensidade de associação foi observada com relação ao tipo de serviço, classe social, estar trabalhando e número de consultas de pré-natal, estando maior escolaridade associada ao serviço privado.

Analisando as variáveis "número de partos anteriores por cesárea" e "opções contraceptivas", elas não mostraram associação estatisticamente significante com a escolaridade. Porém, tais resultados demonstraram a influência da educação configurada por aquelas mulheres do serviço privado, quanto à questão do planejamento familiar, opções contraceptivas e, em última instância, o nivel de fecundidade ${ }^{2,5}$.

Constatou-se, também, que a maioria das mulheres com mais anos de estudo (55\%) expressou o desejo de fazer a esterilização mais cedo e com menos filhos. Para evitar filhos, especialmente entre as primíparas, a maioria revelou utilizar pílula como anticoncepcional.

Tem-se relatado que, muitas vezes, o medo ou o temor do parto normal funcionam como principais fatores socioculturais que levam ao parto cesáreo ${ }^{9,13}$. Faúndes et al. ${ }^{14}$ ressaltam a preferência pela cesárea e vinculam a sua prática à questão da dor, na sua grande maioria. O estudo em análise também encontrou tal afirmação, mas remete esta situação a uma reflexão quando $79 \%$ das mulheres provenientes do SUS são submetidas à ocitocina como forma de intensificar o parto e apenas $16 \%$ tomaram alguma medicação para aliviar a dor na hora do parto, o que torna o parto cesáreo desejado por qualquer mulher. Outra opção pela cesárea é a sua utilização como veículo facilitador para a ligadura de trompas, indicação dificilmente perceptivel, devido às várias formas de mascarar tal realidade.

Quanto à busca pela esterilização, estudos mostraram que a mulher de baixa renda tem necessidade e vontade de controlar sua fertilidade, porém existe dificuldade em adotar métodos reversiveis, devido à falta de planejamento familiar de qualidade, e a esterilização, pela falta de condição de pagar o ato médico ${ }^{5,15}$. O presente estudo reforça também essa condição, o que nos reporta a uma situação de análise que reflete a questão da queda da fecundidade em mulheres com melhor poder aquisitivo e com mais anos de estudo.

Um estudo ${ }^{16}$ com base na PNDS de 1996 revelou que mulheres na faixa etária de 15 a 24, unidas e associadas ao nivel de escolaridade e usuárias de métodos anticoncepcionais, em alguns estados do Nordeste, comparadas entre si, tinham comportamento semelhantes, quando não desejavam querer mais de dois filhos, revelando, portanto, que essas mulheres são as mais necessitadas de meios eficazes para um planejamento familiar ou de um controle saudável no marco da saúde reprodutiva e da educação formal, por se tratar de uma geração que determina a trajetória da reprodução.

De uma forma geral, o SINASC $(2004){ }^{17}$ para Natal mostra que as mulheres ao longo dos anos contribuem para a queda da fecundidade, visto que $48 \%$ tem de 1 a 3 filhos vivos ${ }^{18}$.

Os resultados obtidos ratificam a forte associação entre a educação e diversos fatores como: tipo de parto, número de filhos, número de consultas de pré-natal, dentre outros, apontando a importância de investimento na melhoria da escolaridade das mulheres e em programas que efetivamente possam dar cobertura total com todos os métodos anticoncepcionais disponiveis, em serviços de saúde de planejamento familiar, numa promoção de qualidade, eficiência e eficácia na prestação desses serviços, permitindo atender desta forma a concepção e a contracepção. Portanto, é necessário desmistificar a idéia de que a contracepção com credibilidade é aquela que desfruta a LT, entre as mulheres que desejam evitar engravidar. Contudo, o planejamento familiar deve ser defendido não somente com o objetivo de reduzir a fecundidade dos menos favorecidos, mas como um meio de garantir o pleno exercício dos direitos reprodutivos, ao casal que deseja regular o número de filhos desejados.

Assim, os resultados nos levam a concluir que existe distanciamento muito grande entre o parto desejado e o realizado, quando relacionado com o serviço de atendimento, demonstrando dessa forma, que gestantes mais jovens precisam ser melhor trabalhadas, para mudar a prática obstétrica no Brasil, principalmente entre aquelas atendidas no serviço privado.

\section{Agradecimentos}

Os autores agradecem à Coordenação Geral do Estudo Multicêntrico sobre a Saúde Reprodutiva no Brasil, pela autorização para trabalhar as informações referentes a Natal, no Estado do Rio Grande do Norte, cujos resultados são parcialmente apresentados neste trabalho. 
Esta pesquisa teve o apoio financeiro do Population Research Center (PRC), do National Institutes of Health (NIH) e do Fundo de População das Nações Unidas (UNFPA).

\section{Referências}

1. Bem-Estar Familiar no Brasil (BEMFAM). Pesquisa nacional sobre demografia e saúde. Rio de Janeiro; 1991.

2. Bem-Estar Familiar no Brasil (BEMFAM). Pesquisa nacional sobre demografia e saúde. Rio de Janeiro; 1997.

3. Berquó E. Brasil, um caso exemplar: anticoncepção e parto cirúrgico á espera de uma ação exemplar. São Paulo: Núcleo de Estudos Populacionais (NEPO)/ UNICAMP; 1993.

4. Instituto Brasileiro de Geografia e Estatística (IBGE). Departamento de População e Indicadores Sociais. Pesquisa sobre padrões de vida (1996-1997). Rio de Janeiro; 1998.

5. Perpétuo IHO. Contracepção e declínio da fecundidade na Região Nordeste, 1980-1996. Rev Bras Estud Popul. 1998;15(1):43-56.

6. Instituto Brasileiro de Geografia e Estatística (IBGE). Censo demográfico 1991. Rio de Janeiro; 1994.

7. Michelazzo D, Yaslle MEHD, Mendes MC, Patta MC, Rocha JSY, Moura, MD. Indicadores sociais de grávidas adolescentes: estudo caso-controle. Rev Bras Ginecol Obstet. 2004;26(8):633-9.

8. Cecatti JG, Andreucci CB, Cacheira PS, Pires HMB, Pinto e Silva JL, Aquino MMA. Fatores associados à realização de cesárea em primíparas com uma cesárea anterior. Rev Bras Ginecol Obstet. 2000;22(3):175-9.

9. Tedesco RP, Maia Filho NL, Mathias L, Benez AL, Castro VCL, Bourroul GM, et al. Fatores determinantes para as expectativas de primigestas acerca da via de parto. Rev Bras Ginecol Obstet. 2004;26(10):791-8.

10.Potter JE, Berquo E, Perpetuo IH, Leal OF, Hopkins K, Souza MR, et al. Unwanted caesarean sections among public and private patients in Brazil: prospective study. BMJ. 2001;323(7322):1155-8.

11. Natal. Prefeitura Municipal. Secretaria Municipal de Saúde [sítio na a Internet]. Estatísticas vitais. Sistema de informação em nascidos vivos. 2005 [citado 2005 Out 24 ]. Disponivel em: www.natal.rn.gov.br/

12. Sociedade Brasileira de Pesquisa de Mercado. Novo critério Brasil: mercado volta a ter critério único. Rev SBPM. 1997;1(2):29.

13. Camara MFB, Medeiros M, Barbosa M. Fatores sócio-culturais que influenciam a alta incidência de cesáreas e os vazios da assistência de enfermagem. Rev Eletron Enfermagem [periódico na Internet]. 2000 [citado 2005 Out 24 ];2(2). Disponivel em: http:// wwww.fen.ufg.br/revista

14.Faúndes A, Pádua KS, Osis MJD, Cecatti JG, Sousa, $\mathrm{MH}$. Opinião de mulheres e médicos brasileiros sobre a preferência pela via de parto. Rev Saúde Pública. 2004;38(4):488-94.

15.Vieira EM. A esterilização de mulheres de baixa renda em região metropolitana do sudeste do Brasil e fatores ligados à sua prevalência. Rev Saúde Pública. 1994;28(6):440-8.

16.Aguirre MAC, Freire FHMA, Barbosa LM, Barboza IM, Moraes LL. As preferências reprodutivas no Rio Grande do Norte e estados do nordeste brasileiro. Rev FAPERN. 2005;1(2):14-6.

17. Natal. Prefeitura Municipal. Secretaria Municipal de Saúde [sitio na Internet]. Estatísticas vitais. Sistema de informação em nascidos vivos. 2004 [citado 2006 Jan 10]. Disponivel em: http://www.natal.sms.gov.br

18. Universidade Federal do Rio Grande do Norte (UFRN). Departamento de Estatística. Grupo de Estudos Demográficos. Banco de dados do estudo multicêntrico sobre saúde reprodutiva do Brasil: dados do Rio Grande do Norte. Natal; 2000. 